\title{
Changes in the balance between motor cortical excitation and inhibition in focal, task specific dystonia
}

\author{
M C Ridding, G Sheean, J C Rothwell, R Inzelberg, T Kujirai
}

\begin{abstract}
Transcranial magnetic stimulation has been used in a double pulse paradigm to investigate the excitability of intrinsic motor cortical circuits in 15 patients with focal task specific dystonia of the right hand and a group of eight age matched controls. The left hemisphere was examined in five patients; in the remainder, both hemispheres were tested. There was no significant difference in stimulation threshold between patients and controls nor between the left and right hemispheres in the patients. There was a significant decrease in early corticocortical suppression when comparing stimulation of the left hemisphere in the patients and controls at interstimulus intervals of 1-15 $m s(P<0.01)$. There was no difference in the amount of suppression in the right and left hemispheres of the patients. It is concluded that in focal task specific dystonia there is shift in the balance between excitation and inhibition in local circuits of the motor cortex which leads to a net decrease in the amount of short latency suppression. These changes reflect disturbed basal ganglia input to the motor cortex. Reduced excitability of cortical inhibitory circuits may be one factor which contributes to the excessive and inappropriate muscle contraction which occurs during fine motor tasks in patients with focal dystonia.
\end{abstract}

(F Neurol Neurosurg Psychiatry 1995;59:493-498)

Keywords: dystonia; cortex; inhibition

Focal, task specific, dystonia, of which writer's cramp and musician's dystonia are examples, is characterised by excessive muscular activation during fine manipulative tasks. In the case of writing this excessive activity is usually seen in muscles of the forearm and hand. On the rare occasions when an abnormality can be demonstrated it is usually confined to the putamen, caudate, thalamus, and globus pallidus or their connecting pathways. ${ }^{1}$ Writer's cramp is therefore believed to be a disease of the basal ganglia.

There have been several electrophysiological studies in patients with dystonia. Many of these have focused on spinal or brainstem abnormalities. For example, a reduction in reciprocal inhibition between antagonist forearm muscles has been described in writer's cramp, ${ }^{23}$ and changes in the blink reflex recovery cycle have been seen in patients with blepharospasm and cranial dystonia. ${ }^{4-6}$ More recently, however, several reports have considered the possibility that pathophysiological changes may also occur within the cerebral cortex, particularly in the motor areas which are a primary projection target of the basal ganglia. The Bereitschaftspotential, which is thought to be generated in the supplementary and primary motor areas, is decreased (in its later phase) before self initiated movements. ${ }^{78}$ During free choice joystick movements PET studies have shown increased activity in the rostral supplementary motor area and decreased activity in the caudal supplementary motor area and primary motor cortex. Reilly et $a l^{9}$ reported bilateral abnormalities of the N30 component of the somatosensory evoked potential. Finally, Thompson et al ${ }^{10}$ reported that the duration of the silent period after transcranial magnetic stimulation was in the low normal range.

Recently, we have reported a technique employing double pulse magnetic stimulation which gives an indication of the balance between excitability of local intracortical inhibitory and excitatory neuronal populations. ${ }^{11} \mathrm{~W}$ ith this technique it has been possible to show that the relative excitability of inhibitory circuits is reduced in Parkinson's disease, ${ }^{12}$ in which there is known disease of the basal ganglia. In the present study we have examined intracortical inhibition in the motor cortex of a group of patients with focal task specific dystonia.

\section{Methods}

PATIENTS

With the approval of the local ethics committee we studied 15 patients with focal task specific dystonia (14 cases of writer's cramp and one of simple musician's dystonia), mean age 47 (SD 13) years), and eight neurologically normal age matched controls (49 (SD 14) years). Patients and control subjects gave informed consent. All patients and controls were right handed. Four of the patients with writer's cramp were being treated with botulinum toxin. The patients with writer's cramp were subdivided into two groups depending on their symptoms. If their symptoms were specific to writing and only involved muscles employed for writing they were considered to 
have simple writer's cramp. If their symptoms were specific to writing but also involved muscles not usually employed for writing (more generalised muscle involvement) then they were considered to have dystonic writer's cramp. ${ }^{13}$

All experiments were performed with a figure of eight stimulating coil (external loop diameters $9 \mathrm{~cm}$ ) powered with two high power Magstim 200 magnetic stimulators (Magstim, Dyfed, UK), which were linked via a Bistim module (Magstim, Dyfed, UK). This allowed the delivery of two magnetic pulses through the one coil at short interstimulus intervals. With this technique there is a reduction in the power of the pulses due to the switching characteristics of the Bistim module. This results in the power output being about $30 \%$ lower than that seen on the display of the magnetic stimulators. Throughout this paper the intensities quoted are for stimulation through the Bistim module and, therefore, are some $30 \%$ higher than the true output intensity of the stimulator. The coil was held so that it induced electric current in the motor cortex which flowed in a posterior to anterior direction. Silver/silver chloride surface recording electrodes were used to record evoked responses from the right first dorsal interosseous muscle. The active electrode was placed over the muscle belly and the reference electrode was placed over the second metacarpophalangeal joint. In 10 of the patients responses were also recorded from the left interosseous muscle after stimulation of the right motor cortex. Responses were recorded on to a PC using a 1401 laboratory interface (Cambridge Electronic Design, Cambridge, UK) for "off line" analysis.

\section{THRESHOLDS}

The threshold for evoking responses in the target muscle (first dorsal interosseous muscle) was assessed with single stimuli. The stimuli were still delivered with the Bistim module connected and so the values quoted are about $30 \%$ higher than the values that would have been obtained with a single conventionally set up stimulator. Firstly, the threshold for eliciting responses with the target muscle relaxed was assessed. To help the subject maintain relaxation audiovisual feedback was given. The coil was placed on the optimal scalp location for eliciting responses in the target muscle and stimuli were applied. Initially the stimulus was increased in 5\% steps until an estimation of threshold was obtained. The stimulator output was then adjusted in $1 \%$ steps until an accurate measure of threshold was reached. Threshold was defined as being the stimulus intensity that produced clear EMG responses (of at least 50 $\mu \mathrm{V}$ peak to peak amplitude) in $50 \%$ of successive trials. For the determination of active threshold subjects were instructed to maintain a steady, minimal, background contraction (5-10\% MVC) while the above procedure was repeated. Subjects were again given audiovisual feedback to assist them in maintaining a steady contraction.
PAIRED PULSE SUPPRESSION

This technique is described in detail elsewhere. ${ }^{11}$ Briefly, two magnetic stimuli were given through the same stimulating coil over the motor cortex and the effect of the first (conditioning) stimulus on the second (test) stimulus was investigated. The conditioning stimulus was set at an intensity of $5 \%$ (of stimulator output) below active threshold. The second, test, shock intensity was adjusted to evoke a muscle response in the relaxed first dorsal interosseous muscle with an amplitude of about $1 \mathrm{mV}$ peak to peak. The timing of the conditioning shock was altered in relation to the test shock. Interstimulus intervals between 1 and $15 \mathrm{~ms}$ were investigated. Three blocks of 40 trials were recorded for each subject. Each block consisted of four different conditions; test alone and test + conditioning at three different interstimulus intervals. The order of the presentation was generated pseudorandomly by means of a 1401 laboratory interface (Cambridge Electronic Design, Cambridge, England). For these recordings muscle relaxation is very important and subjects were given audiovisual feedback at high gain to assist in maintaining complete relaxation. If EMG activity became apparent during data collection responses were rejected. Measurements were made on individual responses and the area of the conditioned response, at each interstimulus interval, was expressed as a percentage of the area of the test response alone.

\section{STATISTICAL ANALYSIS}

In the analysis of the experiments we considered the following comparisons: (a) patients' left hemisphere and controls' left hemisphere, (b) patients' right hemisphere and controls' left hemisphere (c) the left and right hemispheres of individual patients (d) those patients who were on botulinum toxin and those on no medication, and (e) the level of inhibition in patients with simple writer's cramp and those patients with dystonic writer's cramp. For the main set of experiments with paired pulses the effect of group (patients/controls), interstimulus intervals, and the interaction between GROUP $\times$ interstimulus intervals were analysed using multivariate analysis of variance (MANOVA). The comparison of the inhibition of the right and left hemisphere in the patients, the effect of botulinum toxin, and the comparison of inhibition in the two subgroups of patients with writer's cramp (simple and dystonic) was performed with MANOVA. For comparison of threshold data in the patients and controls a $t$ test was used. For comparison of right and left hemispheric thresholds in the patients the paired Student's $t$ test was used.

\section{Results}

\section{THRESHOLDS}

In the relaxed condition, after stimulation of the left hemisphere, the mean threshold for the controls was 56 (SD 9)\% and 57 (SD $10) \%$ for the patients. The threshold after 

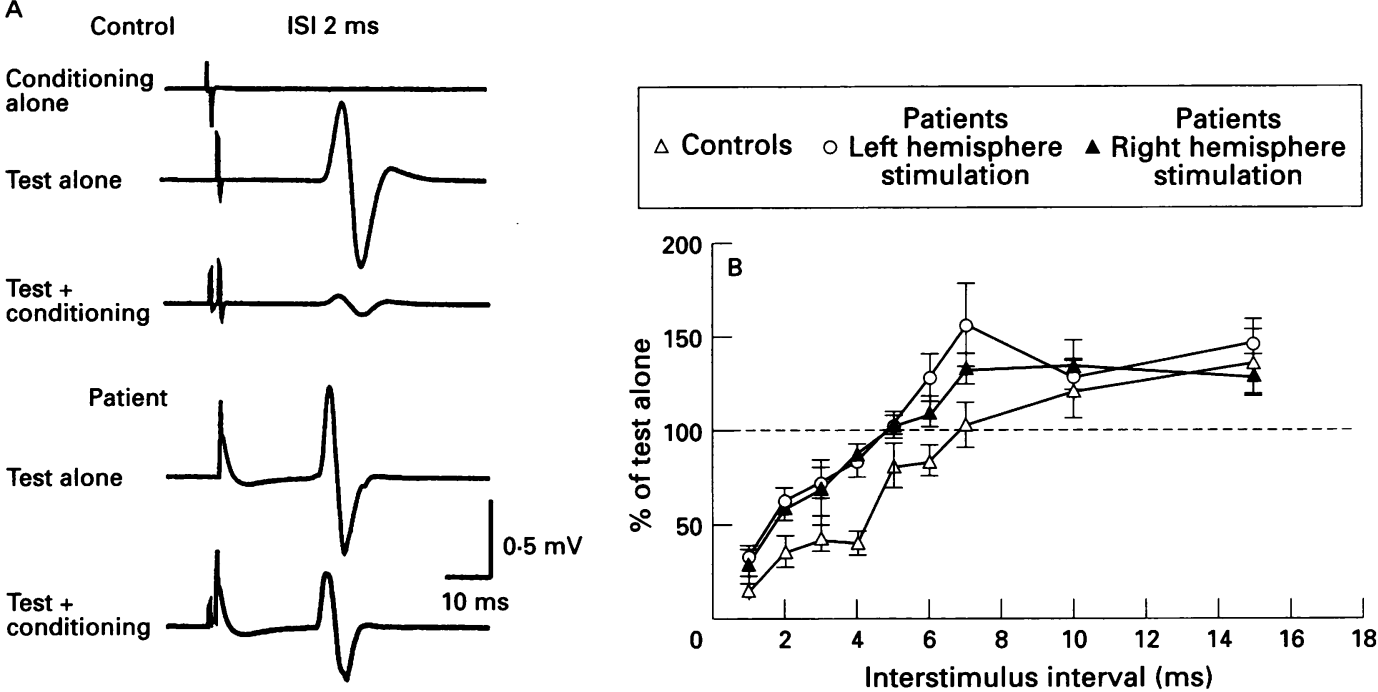

Figure 1 Time course of paired pulse effects. (A) Raw data traces from a control subject (top set of three traces) and a representative patient with writer's cramp (bottom set of two traces). These data were obtained after left hemispheric stimulation and responses recorded in the relaxed right first dorsal interosseous muscle. In both the control subject and the patient the subthreshold conditioning stimulus evoked no response in the target muscle (see top trace), whereas the test stimulus evoked a clear EMG response of approximately $1 \mathrm{mV}$ (peak to peak amplitude). In the control subject when the conditioning stimulus was given $2 \mathrm{~ms}$ before the test stimulus there was clear suppression of the response (bottom trace for the control subject). In the case of the patient there was much less suppression of the test response when conditioned at an interstimulus interval of $2 \mathrm{~ms}$ (see bottom trace). (B) Data obtained across all interstimulus intervals in controls and patients (stimulation of both both left and right hemisphere).

stimulation of the right hemisphere in the patients was 55 (SD 9)\%. When active, after left hemispheric stimulation, the threshold for the controls was 40 (SD 9)\% and for the patients 42 (SD 11)\%. After right hemispheric stimulation the threshold in the patients was 40 (SD 8)\%. There was no significant difference in threshold in either the relaxed or active conditions when comparing left hemispheric stimulation in the patients and controls (relaxed state: $t$ test, $\mathrm{P}>0.5$; active state: $P>0 \cdot 5$ ). Also, no significant difference was seen in the thresholds for stimulation when comparing the right and left hemispheres in the patients (relaxed condition: paired $t$ test, $\mathrm{P}>0.5$; active condition: $\mathrm{P}>0 \cdot 1$ ).

Figure 2 (A) Average
level of inhibition across interstimulus intervals of $1-6 \mathrm{~ms}$ in controls and patients (stimulation of both left and right hemisphere). The $y$-axis is the area of the average conditioned response expressed in terms of the area of the average response to the test stimulus alone. There is significantly less inhibition in the patients after either left MANOVA

$\left.P<0.005^{\star * *}\right)$ or right hemisphere stimulation (MANOVA,

$P<0.05^{* *}$ ). (B) Average facilitation of the test response when preceded by a conditioning stimulus at interstimulus intervals of $7-15 \mathrm{~ms}$. There is no significant difference between the level of facilitation in controls and patients (stimulation of either left or right

hemisphere) (MANOVA $P>0 \cdot 1$.
PAIRED PULSE SUPPRESSION

When the whole time course (interstimulus intervals 1-15 ms) was examined there was a significant difference between the patients' left hemisphere and the control subjects' left hemisphere (MANOVA, $P<0.01$ ). Also, there was a significant difference when comparing the patients' right hemisphere with the control subjects' left hemisphere (MANOVA, $P<0.05)$. In both of these cases there was no significant interaction between group and interstimulus intervals $(P>0.05)$. Figure 1 shows the time course of the inhibition. We know from previous studies and this study that in normal subjects the time course can be divided into an "inhibitory phase" (interstimulus intervals of 1-6 ms) and a "facilitatory phase" (interstimulus intervals of 7-15 ms). On the basis of this division further analysis was performed on the data. When we averaged the data across interstimulus intervals of 1-6 ms - the "inhibitory phase"-there was significantly less inhibition both when comparing the patients' left hemisphere with the control subjects' left hemisphere (MANOVA, $\mathrm{P}<0.001$ ) and also when comparing the patients' right hemisphere with the control subjects' left hemisphere (MANOVA, $P<0.01)$. When we averaged the data across interstimulus intervals of 7-15 ms-the "facilitatory phase"-there was no significant difference comparing either the patients' left hemisphere with the controls' left hemisphere (MANOVA, $P>0.2$ ) or the patients' right hemisphere with the controls' left hemisphere $(P>0.5)$. Figure 2 shows the average level of inhibition and facilitation across these blocks of interstimulus intervals. In the controls, conditioned responses were suppressed to an average of 50 (SD 15)\% of the test response alone across interstimulus intervals of $1-6 \mathrm{~ms}$ 
after left hemispheric stimulation. In the patients, responses were suppressed to only 80 (SD 17)\% after left hemispheric stimulation and 76 (SD 20)\% after right hemispheric stimulation. In the control subjects responses were facilitated to an average of 12 (SD 35)\% of the test response across interstimulus intervals of $7-15 \mathrm{~ms}$, whereas in the patients, responses were facilitated to 144 (SD 44)\% after stimulation of the left hemisphere and 127 (SD 23)\% after stimulation of the right hemisphere. Botulinum toxin had no significant effect on the level of inhibition observed in the left hemisphere of patients (MANOVA, $P>0.05)$. There was no significant difference in the level of inhibition when comparing patients with simple writer's cramp and those with dystonic writer's cramp (MANOVA, $P>0 \cdot 5)$.

\section{Discussion}

Writer's cramp and musician's dystonia are examples of task specific focal dystonias. As a result they are believed to be due to dysfunction of the basal ganglia or their connections. The physiological basis of these conditions has been debated for a long time. As outlined in the introduction, there is evidence of disordered spinal cord and brainstem reflexes in several types of focal dystonia. As no abnormality has ever been found at either of these sites, however, it is usually assumed that the changes are due to an alteration in descending inputs from other, higher, structures. Unfortunately, it is unclear which particular structures might be important in relaying these effects. For example, changes in the blink reflex recovery could be due to descending connections between basal ganglia or brainstem, or due to changes in projections to brainstem from cerebral cortex deprived of its normal input from basal ganglia. Similarly, spinal mechanisms of reciprocal inhibition receive input from many supraspinal structures including both the cortex and brainstem.

The purpose of the present paper was to investigate the physiology of motor cortical areas as these are known to be one of the primary output targets of the basal ganglia. To do this we used the technique of transcranial magnetic stimulation over the motor cortex. Like others we found that the threshold for evoking an EMG response in active or relaxed muscle was the same as in normal subjects. ${ }^{10}$ By contrast, major changes in excitability became evident when we used double pulse testing at short interstimulus intervals. With this technique, a subthreshold conditioning shock is given before a suprathreshold test shock. In control subjects this produces a pronounced suppression of the EMG response to the test shock at interstimulus intervals of 1-6 ms. We have argued previously ${ }^{11}$ that this is of a cortical nature and suggest that it reflects activity of local intracortical inhibitory (probably GABAergic) interneurons. The main reasons for this are (1) the conditioning stimulus is of such a low intensity $(5 \%$ or more below the threshold for evoking EMG responses in preactivated muscle) that it probably does not produce any descending volley in the pyramidal tract; (2) a conditioning stimulus produces greater suppression of responses evoked by a magnetic than an anodal electrical stimulus to the motor cortex. " Because both forms of stimulation are thought to activate the same descending corticospinal pathways, the conditioning shock is unlikely to be acting at a subcortical level. Instead, the differential effect is thought to arise because of differences in the way electric and magnetic stimuli activate the corticospinal system. Anodal electrical stimuli over the scalp tend to activate the pyramidal tract neurons directly whereas magnetic stimuli tend to activate the same neurons transynaptically. ${ }^{14}$ Thus the size of responses evoked by magnetic stimulation should be more affected by changes in cortical excitability than those evoked by anodal stimuli. If the conditioning stimulus affects cortical excitability then this explains why there is a greater suppression of responses to magnetic than electrical stimulation.

PAIRED PULSE SUPPRESSION IN DYSTONIA

The time course of paired pulse testing is complex, beginning with a period of pronounced suppression followed by less prominent facilitation. Many circuits, with overlapping time courses could contribute to the effect. The curve shows only that inhibition dominates the early timing whereas facilitation dominates later. Less suppression early in the time course in the patients with focal dystonia indicates that that there is a relative decrease in the excitability of cortical inhibitory systems.

The question is whether this reduction has any relation to the clinical picture of dystonia. It is interesting to note that in primates injection of the GABA antagonist bicuculline into the motor cortex results in loss of directional specificity of cortical cells ${ }^{15}$ and inappropriate contraction of antagonist muscles during reaction time wrist movements. ${ }^{16}$ We therefore suggest that the reduction in excitability of intracortical inhibitory circuits which we have found in patients with focal dystonia (the activity of which, we suggest, is measured by this paired pulse technique) may be one factor which contributes to the inappropriate and excessive muscle activity which occurs when they attempt fine manipulations. Reduced activity in inhibitory circuits may also explain why PET studies of patients with idiopathic dystonia show a decreased blood flow in the primary motor cortex during voluntary joystick movements. ${ }^{17}$

One criticism of the proposed role of cortical inhibition in producing dystonia is that the reduction in paired pulse suppression is seen both in the motor cortex contralateral to the affected hand and in the other hemisphere (although the second comparison was made between the patients' right hemisphere and the controls' left hemisphere we have not experienced any major variation in the amount of inhibition in the two hemispheres 
of normal subjects). Such bilateral abnormalities in cases of unilateral dystonia ${ }^{918}$ are not an uncommon result. Interestingly, when some patients with writer's cramp learn to write with their "unaffected" hand, because of the difficulties with their dominant hand, as many as $25 \%$ of them go on to develop symptoms in the other hand. ${ }^{13}$ This suggests that subclinical abnormalities may have been present bilaterally from the beginning of the disease.

Another apparent problem with the present findings is that there was no significant difference in the level of inhibition when we compared patients with simple writer's cramp with those with dystonic writer's cramp, even though the dystonic spasms were worse in the second group. This may have been because the muscle investigated (first dorsal interosseous muscle) was equally likely to be affected in either group of patients and hence showed a similar lack of cortical inhibition in the two groups. It may be that if we had investigated muscles that were affected in patients with dystonic writer's cramp but not in the simple group there may have been a difference in the level of inhibition. Unfortunately, it is more difficult to perform these measurements on proximal muscles as the threshold for stimulation is usually much higher than in distal muscles. Also it is important to remember that these recordings were obtained when the subjects were at rest, and had no dystonic symptoms. It is possible that if we had investigated the patients when active a different picture may have emerged.

We conclude that, although it is impossible to be certain, it is likely that these alterations in the excitability of inhibitory systems within the motor cortex play a part in the abnormal movements seen in dystonia.

Four of the patients studied were being treated with botulinum toxin. Botulinum toxin acts principally at the neuromuscular region but may also have an effect on central structures, either by retrograde transmission up motor axons, or by secondary changes in neural circuitry consequent on altered peripheral input caused by muscle weakness. For example, it has been reported that reciprocal inhibition in patients with writer's cramp is normalised after botulinum toxin treatment. ${ }^{19}$ At the level of the cortex, however, recent PET studies have failed to show any significant alterations in the level of cortical blood flow after botulinum toxin treatment. ${ }^{20}$ Similarly, our results showed no significant difference in the level of corticocortical inhibition in those patients who were being treated with botulinum toxin and those who were not. Although only a few patients were studied, this suggests that botulinum toxin has no effect on the activity of local cortical inhibitory circuits.

COMPARISON WITH PREVIOUS RESULTS IN PATIENTS WITH PARKINSON'S DISEASE

We have shown previously that, as in the present results, paired pulse suppression is reduced in patients with Parkinson's disease. ${ }^{12}$
Close inspection of the data in the two groups suggests that there may be subtle differences in the form of the suppression in Parkinson's disease and dystonia. The latter seem to have reduced suppression at all intervals, whereas the former show more reduction at specific timings $(2,4$, and $5 \mathrm{~ms})$. It is, however, probably inapropriate to speculate on such differences until larger numbers of patients are studied. It is interesting to note, though, that although the lack of suppression occurs in both these instances of basal ganglia disease, suppression is normal in patients with cerebellar deficits. ${ }^{21}$

Finally, the question arises as to why the decreased cortical inhibition produces excessive muscular activity in patients with dystonia, but not in patients with Parkinson's disease. One possibility is that the movement command itself is reduced in Parkinson's disease (resulting in bradykinesia) and the effects of reduced cortical inhibition are only apparent in the presence of a relatively normal movement command. Hence the effects on cortical inhibition are masked in untreated Parkinson's disease. When the movement command is restored, as during drug induced dyskinesiae, the resulting excess muscle activity becomes clear.

In conclusion, we have shown abnormalities of inhibition in the motor cortex of patients with two manifestations of task specific focal dystonia-namely, writer's cramp and musician's dystonia. These abnormalities are seen bilaterally and are not confined to the motor cortex projecting to the affected limb. Disease in the basal ganglia may affect inhibition in the bilateral areas of motor cortex but only leads to symptoms with the repeated performance of skilled manipulative tasks.

MCR was supported by Action Research. RI was supported by the British Council and the Clore Foundation.

1 Rothwell JC, Obeso JA. The anatomical and physiological basis of torsion dystonia. In: Movement disorders 2 . London: Butterworths, 1987:313-31.

2 Panizza ME, Hallett $M$, Nilsson J. Reciprocal inhibition in patients with hand cramps. Neurology 1989;39:85-9.

3 Nakashima K, Rothwell JC, Day BL, Thompson PD, Shannon K, Marsden CD. Reciprocal inhibition between forearm muscles in patients with writer's cramp between forearm muscles in patients with writer's cramp
and other occupational cramps, symptomatic hemidysand other occupational cramps, symptomatic hemidys-
tonia and hemiparesis due to stroke. Brain 1989; tonia and hem

4 Beradelli A, Rothwell JC, Day BL, Marsden CD. Pathophysiology of blepharospasm and oromandibular dystonia. Brain 1985;108:593-608.

5 Tolosa E, Montserrat L, Bayes A. Blink reflex studies in focal dystonias: enhanced excitability of brainstem interneurones in cranial dystonia and spasmodic torticollis. Mov Dis 1988;3:61-9.

6 Nakashima K, Rothwell JC, Thompson PD, et al. The blink reflex in patients with idiopathic torsion dystonia. Arch Neurol 1990;47:413-6.

7 Feve A, Bathien N, Rondot P. Abnormal movement related potentials in patients with lesions of the basal ganglia and anterior thalamus. $f$ Neurol Neurosurg ganglia and anterior that
Psychiatry 1994;57:100-4.

8 Van Der Kamp W, Rothwell JC, Thompson PD, Day BL, Marsden CD. The movement related potential is abnormal in patients with idiopathic torsion dystonia. Mov Dis 1995 (in press).

9 Reilly JA, Hallett M, Cohen LG, Tarkka IM, Dang N. The N30 component of somatosensory evoked potentials in patients with dystonia. Electroencephalogr Clin Neurophysiol 1992;84:243-47.

10 Thompson ML, Wilson SA, Thickbroom GW, Laing BA, Stell R, Mastaglia FL. Cortical excitability and topography of excitatory and inhibitory areas in focal dystonia. Mov Dis 1994;9(suppl 1):204. 
11 Kujirai T, Caramia MD, Rothwell JC, et al. Corticocortical inhibition in the human motor cortex. $f$ Physiol 1993;471:501-19.

12 Ridding $M C$, Inzelberg $R$, Rothwell JC. Changes in excitability of motor cortical circuitry in patients with excitability of motor cortical circuitry in patient
Parkinson's disease. Ann Neurol 1995;37:181-8.

13 Sheehy MP, Rothwell JC, Marsden CD. Writer's cramp. Adv Neurol 1988;50:457-72.

14 Day BL, Dressler D, Maertens De Noordhout A, et al. Electric and magnetic stimulation of human motor cortex: surface EMG and single motor unit responses. $\mathcal{f}$ Physiol 1989;412:449-73.

15 Matsumura M, Sawaguchi T, Kubota K. GABAergic inhibition of neuronal activity in the primate motor and premotor cortex during voluntary movement. $\mathcal{F}$ Neurophysiol 1992;68:692-702.

16 Matsumura M, Sawaguchi T, Oishi T, Ueki K, Kubota K. Behavioral deficits induced by local injection of bicuculline and muscimol into the primate motor and premotor cortex. F Neurophysiol 1991;65:1542-53.

17 Brooks DJ. Functional imaging studies of movement Move Dis 1994;9(suppl 1):M36.

18 Tempel LW, Perlmutter JS. Abnormal cortical responses in patients with writer's cramp. Neurology 1993;43:2252-7.

19 Priori A, Berardelli A, Mercuri B, Manfredi M. Physiological effects produced by botulinum toxin treatment of upper limb dystonia: changes in reciprocal inhibition between forearm muscles. Brain 1995;118:801-8.

20 Ceballos-Baumann AO, Sheean G, Passingham RE Marsden CD, Brooks DJ. Cerebral activation with stereotyped writing in patients with writer's cramp before and after botulinum toxin treatment: a PET study. Neurology 1995;45(suppl 4):A393.

21 Ugawa Y, Hanajima R, Kanazawa I. Motor cortex inhibition in patients with ataxia. Electroencephalogr Clin Neurophysiol 1994;93:225-9. 